Chinese Journal of Organic Chemistry

NOTE

\title{
砂贝母中异甾体生物碱类成分的研究
}

\author{
黄金昌卉 $a$ 雷 春 ${ }^{\dagger}, a$ 阿吉艾克拜尔・艾萨 $b$ 俞媚华 ${ }^{a}$ \\ 阿布力米提・伊力*,b侯爱君*,a \\ $\left({ }^{a}\right.$ 复旦大学药学院 生药学教研室 上海 201203) \\ $\left({ }^{b}\right.$ 中国科学院新疆理化技术研究所 中国科学院干早区植物资源化学重点实验室 \\ 新疆特有药用资源利用重点实验室 乌鲁木齐 830011)
}

\begin{abstract}
摘要 利用各种柱色谱和高效液相色谱等分离纯化方法, 从砂贝母鳞茎中分离得到 6 个异甾体生物碱类化合物. 根据 质谱、一维/二维核磁共振谱和 $\mathrm{X}$ 射线单晶衍射等技术鉴定了它们的结构, 分别为 karelinine (1), 5-epikarelinine (2), 27-epiebeienine (3), ebeienine (4), persicanidine B (5)和 heilonine (6), 其中化合物 $\mathbf{1} \sim 3$ 为新化合物. 化合物 1 是贝母属中 罕见的 $5 \beta$-jervine 型异甾体生物碱, 具有 $\mathrm{A} / \mathrm{B}$ 环顺式稠合方式. 化合物 $\mathbf{1}$ 和 $\mathbf{2}$ 也是贝母属中首次发现的具有 $15 \alpha-\mathrm{OH}$ 取 代的 jervine 型生物碱.
\end{abstract}

关键词＼cjkstart砂贝母; 异甾体生物碱; karelinine; 5-epikarelinine; 27-epiebeienine

\section{Isosteroidal Alkaloids from Fritillaria karelinii}

\author{
Huang, Jinchang ${ }^{\dagger, a} \quad$ Lei, Chun ${ }^{\dagger, a} \quad$ Aisa, Haji Akber ${ }^{b} \quad$ Yu, Meihua $^{a} \quad$ Yili, Abulimiti*,b $^{*}$ \\ Hou, Aijun *,a \\ ( ${ }^{a}$ Department of Pharmacognosy, School of Pharmacy, Fudan University, Shanghai 201203) \\ ( ${ }^{b}$ Key Laboratory of Plant Resources and Chemistry in Arid Zone and State Key Laboratory Basis of Xinjiang Indigenous \\ Medicinal Plants Resource Utilization, Xinjiang Technical Institute of Physics and Chemistry, \\ Chinese Academy of Sciences, Urumqi 830011)
}

\begin{abstract}
Three new isosteroidal alkaloids, karelinine (1), 5-epikarelinine (2), and 27-epiebeienine (3), were isolated from the bulbs of Fritillaria karelinii by column chromatography and semipreparative HPLC (high performance liquid chromatography), together with three known ones, ebeienine (4), persicanidine B (5), and heilonine (6). Their structures were identified by MS, $1 \mathrm{D} / 2 \mathrm{D}$ NMR, and single-crystal X-ray diffraction. Compound $\mathbf{1}$ is a $5 \beta$-jervine isosteroidal alkaloid featuring a cis-fused A/B ring moiety, rarely found in the Fritillaria genus. Compounds $\mathbf{1}$ and $\mathbf{2}$ also represent the first jervine alkaloids with a $15 \alpha$-hydroxy group from this genus.
\end{abstract}

Keywords Fritillaria karelinii; isosteroidal alkaloids; karelinine; 5-epikarelinine; 27-epiebeienine

贝母为常用中药, 系百合科 (Liliaceae) 贝母属 (Fritillaria) 多种贝母的鳞茎入药, 在我国药用历史悠久, 具有止咳化痰、清热润肺的功效 ${ }^{[1,2]}$. 已有研究表明, 贝 母属植物中含有甾体和异甾体生物碱、甾体㿝苷、二萜、 三萜等成分 ${ }^{[3 \sim 9]}$, 其中异甾体生物碱被认为是贝母属植 物的主要活性成分 ${ }^{[6,10]}$. 砂贝母 (Fritillaria karelinii Baker)主产于新疆西北部地区, 生长在戈壁滩的砂粒
中 $^{[11]}$. 作为新疆特有品种, 砂贝母在维吾尔医药中长期 应用, 具有与贝母相似的功效. 关于砂贝母化学成分研 究鲜有报道, 仅有早期文献报道其含有异甾体生物碱西 贝素 ${ }^{[12]}$. 因此, 为进一步认识砂贝母中的化学成分, 为 其开发利用奠定基础, 本文对采自新疆伊犁地区砂贝母 中的生物碱成分进行了研究. 从其鳞茎中分离得到 6 个 异甾体类生物碱(图 1), 包括 3 个新化合物( $1 \sim 3$ )和 3 个

\footnotetext{
* Corresponding authors. E-mail: abu@ms.xjb.ac.cn; ajhou@shmu.edu.cn

Received September 12, 2018; revised October 19, 2018; published online November 25, 2018.

Project supported by the National Natural Science Foundation of China and the People's Government of Xinjiang Uygur Autonomous Region (NSFC-Xinjiang Joint Fund, No. U1403201).

国家自然科学基金新疆联合基金(No. U1403201)资助项目.

$\dagger$ 共同第一作者(These authors contributed equally to this work).
} 
已知化合物 $(\mathbf{4} \sim \mathbf{6})$, 新化合物命名为 karelinine (1), 5-epikarelinine (2) 和 27-epiebeienine (3). 化合物 1 是贝 母属中罕见的 $5 \beta$-jervine 型异甾体生物碱, 具有 $\mathrm{A} / \mathrm{B}$ 环 顺式稠合方式. 化合物 $\mathbf{1}$ 和 $\mathbf{2}$ 也是贝母属中首次发现具 有 $15 \alpha-\mathrm{OH}$ 取代的 jervine 型生物碱. 化合物 3 是少见的 具有 $\Delta^{13(17)}$ 双键的 cevanine 型异甾体生物碱. 已知化合 物 ebeienine (4)、 persicanidine B (5) 和 heilonine (6)均为 首次从该植物中分离得到。
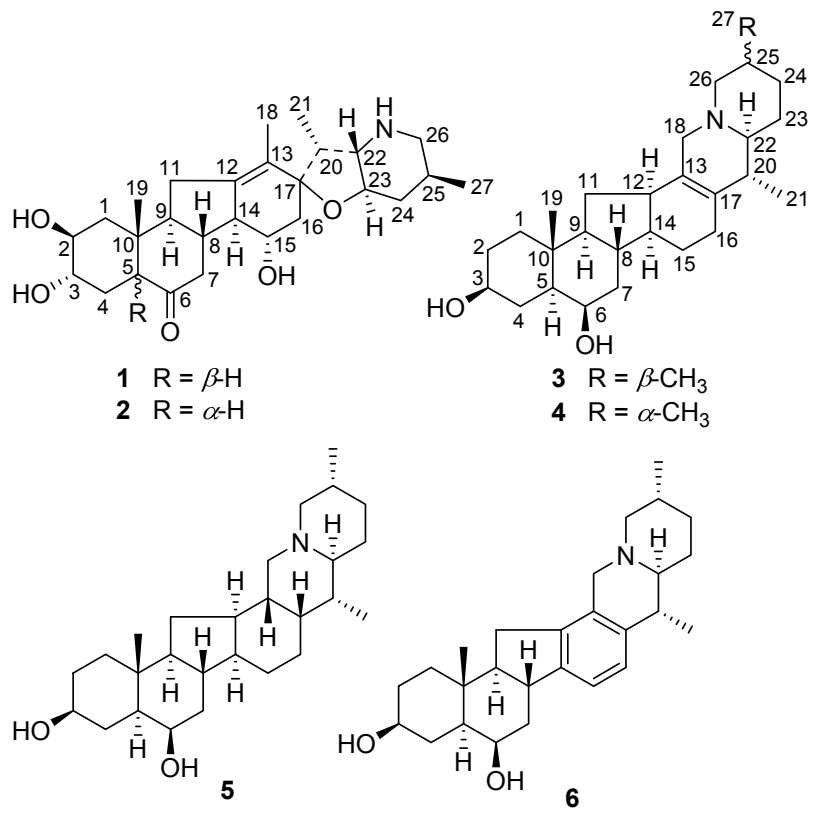

图 1 化合物 $1 \sim 6$ 的结构

Figure 1 Structures of compounds $1 \sim 6$

\section{1 结果与讨论}

化合物 1 为无色晶体，易溶于甲醇. 根据 HRESIMS (high resolution electrospray ionization mass spectroscopy) $\left(\mathrm{m} / \mathrm{z} 460.3057[\mathrm{M}+\mathrm{H}]^{+}\right.$, 计算值为 460.3058) 和 ${ }^{13} \mathrm{C} \mathrm{NMR}$ (nuclear magnetic resonance)数据(表 1), 推断化合物 $\mathbf{1}$ 的 分子式为 $\mathrm{C}_{27} \mathrm{H}_{41} \mathrm{NO}_{5}$, 具有 8 个不饱和度. IR (infrared radiation)光谱显示分子中存在羟基 $\left(3398 \mathrm{~cm}^{-1}\right.$ )和羰基 $\left(1698 \mathrm{~cm}^{-1}\right.$ )官能团. 分析其 ${ }^{1} \mathrm{H} N M R$ 数据(表 1), 可知化 合物 1 的结构中存在 2 个单峰甲基信号 $\left(\delta_{\mathrm{H}} 1.65, \mathrm{~s}\right.$, Me-18; 0.86, s, Me-19), 2 个双峰甲基信号 $\left(\delta_{\mathrm{H}} 0.99, \mathrm{~d}, J=\right.$ $7.4 \mathrm{~Hz}, \mathrm{Me}-21 ; 0.97, \mathrm{~d}, J=6.6 \mathrm{~Hz}, \mathrm{Me}-27)$, 以及 4 个连氧 次甲基信号 $\left(\delta_{\mathrm{H}} 3.65\right.$, ddd, $J=12.6,9.1,4.3 \mathrm{~Hz}, \mathrm{H}-2 ; 3.58$, ddd, $J=12.6,9.5,3.1 \mathrm{~Hz}, \mathrm{H}-15 ; 3.36$, td, $J=11.4,3.6 \mathrm{~Hz}$, $\mathrm{H}-23 ; 3.30 \sim 3.36, \mathrm{~m}, \mathrm{H}-3$ ). 其 ${ }^{13} \mathrm{C}$ NMR 和 DEPT (distortionless enhancement by polarization transfer)数据(表 1)显示该化合物有 27 个碳信号, 可归属为 1 个羰基碳 $\left(\delta_{\mathrm{C}} 216.2, \mathrm{C}-6\right), 2$ 个烯碳 $\left(\delta_{\mathrm{C}} 139.4, \mathrm{C}-12 ; 129.6, \mathrm{C}-13\right), 2$ 个季碳 $\left(\delta_{\mathrm{C}} 89.9, \mathrm{C}-17 ; 39.0, \mathrm{C}-10\right), 11$ 个次甲基[包括 4 个
含氧次甲基 $\left(\delta_{\mathrm{C}} 76.3, \mathrm{C}-23 ; 76.0, \mathrm{C}-3 ; 72.1, \mathrm{C}-15 ; 71.6\right.$, $\mathrm{C}-2)$ 和 1 个连氮次甲基 $\left.\left(\delta_{\mathrm{C}} 66.8, \mathrm{C}-22\right)\right], 7$ 个亚甲基[包括 1 个连氮亚甲基 $\left.\left(\delta_{\mathrm{C}} 55.0, \mathrm{C}-26\right)\right]$, 以及 4 个甲基信号. 根 据以上碳信号和 8 个不饱和度, 推测该化合物为具有 6 个环的甾体类生物碱.

通过分析化合物 1 的 HSQC (heteronuclear single quantum coherence)、 ${ }^{1} \mathrm{H}-{ }^{1} \mathrm{H}$ COSY (correlation spectroscopy)和 HMBC (heteronuclear multiple bond correlation) 数据(图 2)确定了分子的平面结构并归属了碳氢信号. ${ }^{1} \mathrm{H}-{ }^{1} \mathrm{H}$ COSY 谱图中的质子自旋系统 $\mathrm{H}_{2}(1)-\mathrm{H}(2)-\mathrm{H}(3)-$ $\mathrm{H}_{2}(4)-\mathrm{H}(5), \mathrm{H}_{2}(7)-\mathrm{H}(8)-\mathrm{H}(9)-\mathrm{H}_{2}(11)$ 和 $\mathrm{H}(8)-\mathrm{H}(14)-\mathrm{H}(15)-$ $\mathrm{H}_{2}(16)$, 结合以下几组关键的 HMBC 相关: $\mathrm{H}_{3}-19 / \mathrm{C}-1,5$, 9,10; H-5/C-4,6,10; $\mathrm{H}_{2}-7 / \mathrm{C}-6,8,14 ; \mathrm{H}_{2}-16 / \mathrm{C}-13,17 ; \mathrm{H}_{3}-18 /$ C-12,13,17; 以及 $\mathrm{H}-9, \mathrm{H}_{2}-11, \mathrm{H}-14 / \mathrm{C}-12$, 建立了化合物 1 的 6/6/5/6 碳环系统(A/B/C/D 环). ${ }^{1} \mathrm{H}-{ }^{1} \mathrm{H}$ COSY 中的质 子自旋体系 $\mathrm{H}_{3}(21)-\mathrm{H}(20)-\mathrm{H}(22)-\mathrm{H}(23)-\mathrm{H}_{2}(24)-\mathrm{H}(25)-$ $\mathrm{H}_{2}(26)$ 和 $\mathrm{H}(25)-\mathrm{H}_{3}(27)$, 结合 $\mathrm{H}-22 / \mathrm{C}-26$ 的 HMBC 相关, 确定了该化合物存在 1 个 6 元氮杂环 (F环). 根据化合物 1 具有 6 个环，以及 $\mathrm{H}_{2}-16$ 和 $\mathrm{H}_{3}-21$ 同时与含氧季碳 C-17 具有 $\mathrm{HMBC}$ 相关, 推测该化合物在 C-17 和含氧次甲基 C-23 位之间形成了呋喃环(E 环). 由此确定了化合物 1 的平面结构如图 2 所示, 为具有 jervine 骨架的异甾体生 物碱 ${ }^{[5]}$.

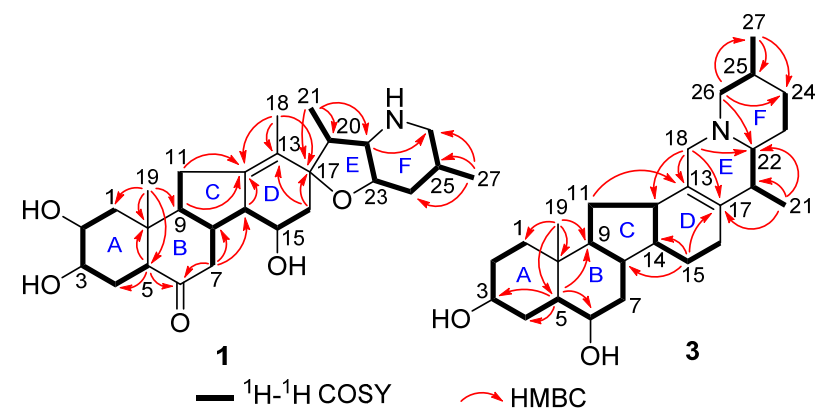

图 2 化合物 $\mathbf{1}$ 和 3 的关键 ${ }^{1} \mathrm{H}-{ }^{1} \mathrm{H}$ COSY 和 $\mathrm{HMBC}$ 相关 Figure 2 Key ${ }^{1} \mathrm{H}-{ }^{1} \mathrm{H}$ COSY and HMBC correlations of compounds $\mathbf{1}$ and $\mathbf{3}$

通过分析化合物 1 的 NOESY (nuclear Overhauser effect spectroscopy)相关(图 3) 和 ${ }^{1} \mathrm{H}$ NMR 数据确定了其 相对构型. 在 NOESY 谱中, H-5 与 H-1a, H-3, Me-19 相 关, 同时 H-3 与 H-1a 相关, 说明 H-3, H-5 与 Me-19 位 于环的同侧, 均为 $\beta$ 取向; H-2 与 H-4b, H-9 有相关, 说 明三者共平面, 为 $\alpha$ 取向. ${ }^{1} \mathrm{H}$ NMR 中, $\mathrm{H}-2$ 与 $\mathrm{H}-1 \mathrm{a}$ 的偶 合常数 $(J=12.6 \mathrm{~Hz})$ 以及 $\mathrm{H}-3$ 的半峰宽值 $\left(W_{1 / 2}=21.0\right.$ $\mathrm{Hz}$ )进一步确定 H-2 和 H-3 均位于 A 环椅式构象的直立 键 ${ }^{[13]}$, 分别取 $\alpha$ 和 $\beta$ 方向. 因此, $\mathrm{A} / \mathrm{B}$ 环具有顺式稠合方 式. H-8 与 H-15, Me-19 有 NOESY 相关, 说明 H-15 与 $\mathrm{H}-8$ 同面, 为 $\beta$ 取向. H-22 与 Me-18, H-20, H-24a, H-26a 


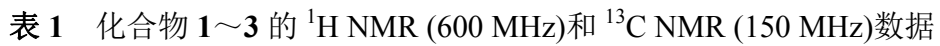

Table $1{ }^{1} \mathrm{H}$ NMR $(600 \mathrm{MHz})$ and ${ }^{13} \mathrm{C}$ NMR $(150 \mathrm{MHz})$ data of compounds $\mathbf{1} \sim \mathbf{3}$

\begin{tabular}{|c|c|c|c|c|c|c|}
\hline \multirow{2}{*}{ No. } & \multicolumn{2}{|l|}{$\mathbf{1}^{a}$} & \multicolumn{2}{|l|}{$2^{a}$} & \multicolumn{2}{|l|}{$3^{b}$} \\
\hline & $\delta_{\mathrm{H}}(J$ in $\mathrm{Hz})$ & $\delta_{\mathrm{C}}$ & $\delta_{\mathrm{H}}(J$ in $\mathrm{Hz})$ & $\delta_{\mathrm{C}}$ & $\delta_{\mathrm{H}}(J$ in $\mathrm{Hz})$ & $\delta_{\mathrm{C}}$ \\
\hline 1 & a $1.18 \mathrm{t}(12.6)$, b $1.92 \sim 1.95 \mathrm{~m}$ & 45.0 & $\begin{array}{l}\text { a } 1.62 \sim 1.66^{c}, \text { b } 1.80 \mathrm{dd} \\
(14.3,3.5)\end{array}$ & 40.6 & $\begin{array}{l}\text { a } 1.04 \sim 1.10^{c}, \text { b } 1.47 \sim \\
1.54^{c}\end{array}$ & 39.6 \\
\hline 2 & 3.65 ddd $(12.6,9.1,4.3)$ & 71.6 & 3.81 br s & 70.9 & $\begin{array}{l}\text { a } 1.42 \sim 1.47 \mathrm{~m}, \mathrm{~b} 1.79 \sim \\
1.84^{c}\end{array}$ & 31.5 \\
\hline 3 & $3.30 \sim 3.36 \mathrm{~m}$ & 76.0 & $3.88 \mathrm{~d}(2.2)$ & 70.7 & $3.58 \sim 3.70 \mathrm{~m}$ & 72.1 \\
\hline 4 & a $1.78 \mathrm{dt}(13.0,4.4)$, b $1.86 \sim 1.92 \mathrm{~m}$ & 34.7 & $\begin{array}{l}\text { a } 1.63 \sim 1.66^{c}, \text { b } 1.86 \sim \\
1.93^{c}\end{array}$ & 24.3 & a $1.64 \sim 1.68 \mathrm{~m}$ & 34.9 \\
\hline 5 & $2.21 \mathrm{dd}(13.0,4.4)$ & 59.8 & $2.77 \sim 2.82 \mathrm{~m}$ & 52.7 & $1.10 \sim 1.14 \mathrm{~m}$ & 47.9 \\
\hline 6 & & 216.2 & & 215.6 & 3.85 br s & 73.3 \\
\hline 7 & a $2.56 \mathrm{t}(13.0)$, b $2.72 \mathrm{dd}(13.0,4.4)$ & 45.1 & $\begin{array}{l}\text { a } 2.42 \text { t (13.1), b } 2.78 \text { dd } \\
(13.1,4.8)\end{array}$ & 48.7 & $\begin{array}{l}\text { a } 1.31 \mathrm{td}(13.5,2.9), \text { b } \\
2.01 \mathrm{dt}(13.5,2.9)\end{array}$ & 39.4 \\
\hline 8 & $1.63 \sim 1.66 \mathrm{~m}$ & 46.9 & $1.66 \sim 1.71^{c}$ & 47.1 & $1.58 \sim 1.64^{c}$ & 40.3 \\
\hline 9 & $2.36 \sim 2.41^{c}$ & 43.8 & $1.91 \sim 1.97^{c}$ & 56.1 & $0.95 \sim 1.03 \mathrm{~m}$ & 56.9 \\
\hline 10 & & 39.0 & & 39.5 & & 35.6 \\
\hline 11 & a $2.00 \sim 2.08^{c}$, b $2.38 \sim 2.44^{c}$ & 29.6 & $\begin{array}{l}\text { a } 1.98 \sim 2.05^{c}, \text { b } 2.35 \mathrm{dd} \\
(16.6,7.7)\end{array}$ & 29.7 & $\begin{array}{l}\text { a } 1.04 \sim 1.10^{c}, \text { b } 1.78 \sim \\
1.84^{c}\end{array}$ & 31.2 \\
\hline 12 & & 139.4 & & 140.0 & $2.29 \mathrm{q}(8.4)$ & 39.4 \\
\hline 13 & & 129.6 & & 129.4 & & 128.2 \\
\hline 14 & $2.00 \sim 2.05^{c}$ & 56.7 & $1.98 \sim 2.05^{c}$ & 56.5 & $1.58 \sim 1.64^{c}$ & 41.3 \\
\hline 15 & $3.58 \mathrm{ddd}(12.6,9.5,3.1)$ & 72.1 & 3.58 ddd $(12.6,9.7,2.9)$ & 72.2 & $\begin{array}{l}\text { a } 1.22 \sim 1.28 \mathrm{~m}, \text { b } 1.68 \sim \\
1.74^{c}\end{array}$ & 27.8 \\
\hline 16 & a $1.61 \mathrm{t}(12.6)$, b $1.97 \mathrm{dd}(12.6,3.1)$ & 42.6 & $\begin{array}{l}\text { a } 1.60 \mathrm{t}(12.6), \mathrm{b} 1.95 \sim \\
1.99^{c}\end{array}$ & 42.7 & $\begin{array}{l}\text { a } 1.68 \sim 1.74^{c}, \text { b } 1.86 \sim \\
1.92^{c}\end{array}$ & 25.7 \\
\hline 17 & & 89.9 & & 90.0 & & 130.8 \\
\hline 18 & $1.65 \mathrm{~s}$ & 13.3 & $1.63 \mathrm{~s}$ & 13.3 & a 2.72 br s, b 2.98 d (15.7) & 57.2 \\
\hline 19 & $0.86 \mathrm{~s}$ & 22.9 & $0.93 \mathrm{~s}$ & 14.8 & $0.94 \mathrm{~s}$ & 15.0 \\
\hline 20 & 2.48 quintet (7.4) & 40.9 & 2.47 quintet (7.4) & 40.9 & $1.95 \sim 1.99 \mathrm{~m}$ & 37.1 \\
\hline 21 & $0.99 \mathrm{~d}(7.4)$ & 11.2 & $0.98 \mathrm{~d}(7.4)$ & 11.2 & $0.92 \mathrm{~d}(6.7)$ & 16.3 \\
\hline 22 & $2.66 \mathrm{t}(9.4)$ & 66.8 & $2.66 \mathrm{t}(9.2)$ & 66.9 & $1.84 \sim 1.90^{c}$ & 64.3 \\
\hline 23 & $3.36 \operatorname{td}(11.4,3.6)$ & 76.3 & $3.36 \operatorname{td}(11.4,3.6)$ & 76.3 & $\begin{array}{l}\text { a } 1.47 \sim 1.54^{c}, \text { b } 1.74 \sim \\
1.78 \mathrm{~m}\end{array}$ & 26.3 \\
\hline 24 & a $1.16 \mathrm{q}(11.4), \mathrm{b} 2.14 \mathrm{dt}(11.4,3.6)$ & 39.8 & $\begin{array}{l}\text { a } 1.16 \text { q (11.4), b } 2.13 \text { br d } \\
(11.4)\end{array}$ & 39.8 & $\begin{array}{l}\text { a } 1.33 \sim 1.39 \mathrm{~m}, \mathrm{~b} 1.47 \sim \\
1.54^{c}\end{array}$ & 29.2 \\
\hline 25 & $1.67 \sim 1.71 \mathrm{~m}$ & 31.9 & $1.66 \sim 1.73^{c}$ & 31.9 & $1.84 \sim 1.88^{c}$ & 29.2 \\
\hline 26 & a $2.31 \mathrm{t}(12.9)$, b $3.05 \mathrm{dd}(12.9,3.9)$ & 55.0 & $\begin{array}{l}\text { a } 2.30 \mathrm{t}(12.9) \\
\text { b } 3.04 \mathrm{dd}(12.9,3.7)\end{array}$ & 55.1 & $\begin{array}{l}\text { a } 2.21 \text { br d (10.9), b } 2.56 \\
\text { dd }(10.9,4.2)\end{array}$ & 60.0 \\
\hline 27 & $0.97 \mathrm{~d}(6.6)$ & 19.2 & $0.96 \mathrm{~d}(6.6)$ & 19.2 & $1.04 \mathrm{~d}(7.0)$ & 18.8 \\
\hline
\end{tabular}

${ }^{a}$ Data were measured in $\mathrm{CD}_{3} \mathrm{OD} .{ }^{b}$ Data were measured in $\mathrm{CDCl}_{3} .{ }^{c}$ Signals are overlapped.
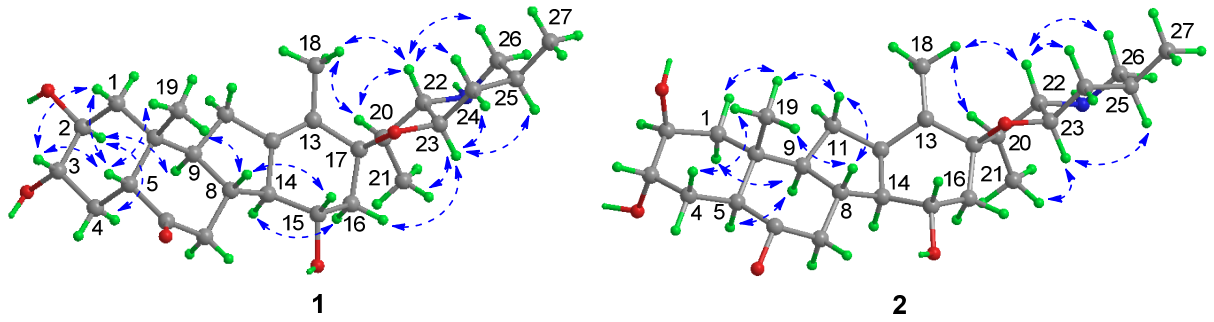

图 3 化合物 $\mathbf{1}$ 和 $\mathbf{2}$ 主要的 NOESY 相关

Figure 3 Key NOESY correlations of compounds 1 and $\mathbf{2}$

的 NOESY 相关, H-23 与 Me-21, H-16b,H-24b, H-25 的 NOESY 相关, 说明 H-22 和 Me-27 为 $\beta$ 取向, H-23 为 $\alpha$
取向, $\mathrm{E} / \mathrm{F}$ 环为反式稠合. $\mathrm{X}$ 射线单晶衍射实验最终确证 了化合物 1 的结构及绝对构型(图 4), 并被命名为 kare- 
linine.

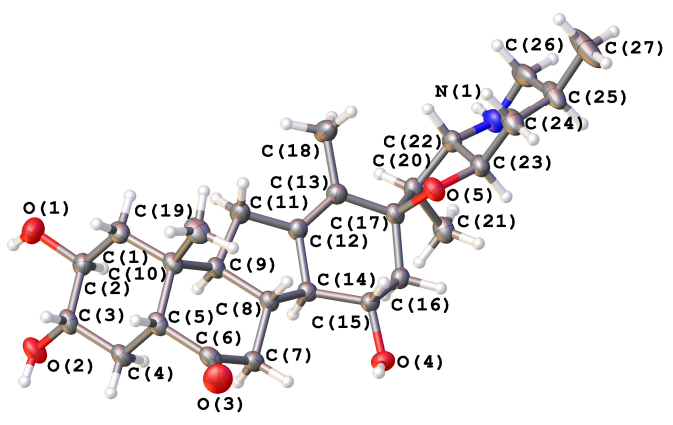

1

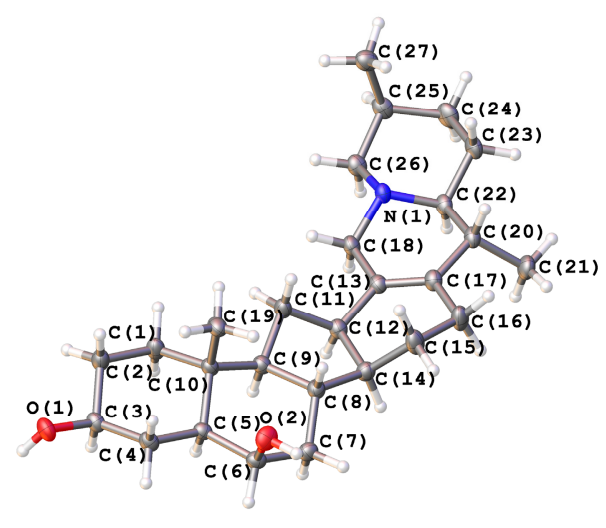

3

图 4 化合物 1 和 3 的 $X$ 射线单晶衍射图

Figure 4 X-ray single-crystal plots of compounds $\mathbf{1}$ and $\mathbf{3}$

化合物 2 为白色无定形粉末, 易溶于甲醇. HRESIMS $\left(\mathrm{m} / \mathrm{z} 460.3048[\mathrm{M}+\mathrm{H}]^{+}\right.$, 计算值为 460.3058) 显示其具有 和化合物 $\mathbf{1}$ 相同的分子式 $\mathrm{C}_{27} \mathrm{H}_{41} \mathrm{NO}_{5}$. 比较化合物 $\mathbf{2}$ 和 $\mathbf{1}$ 的 1D NMR 数据(表 1), 发现它们具有相同的 $C \sim F$ 环结 构, 区别在于 $\mathrm{A} / \mathrm{B}$ 环信号. 根据化合物 $\mathbf{2}$ 的 ${ }^{1} \mathrm{H}-{ }^{1} \mathrm{H} \operatorname{COSY}$ 和 $\mathrm{HMBC}$ 相关谱, 确定其平面结构与化合物 $\mathbf{1}$ 一致. 经 过文献对比发现, 化合物 2 与 $2 \beta, 3 \alpha$-dihydroxy- $5 \alpha$-cholestan-6-one A/B 环的核磁数据极为相似 ${ }^{[14]}$, 推测化合物 2 具有与其相同的 $\mathrm{A} / \mathrm{B}$ 环结构单元, 即化合物 $\mathbf{2}$ 与 $\mathbf{1}$ 相 比, 可能区别仅在于 $\mathrm{A} / \mathrm{B}$ 环为反式稠合. 分析化合物 2 的 ${ }^{1} \mathrm{H}$ NMR 数据(表 1$)$, 两个连氧次甲基质子 $\mathrm{H}-2\left(\delta_{\mathrm{H}}\right.$ $3.81, \mathrm{br} \mathrm{s})$ 和 $\mathrm{H}-3\left(\delta_{\mathrm{H}} 3.88, \mathrm{~d}, J=2.2 \mathrm{~Hz}\right)$ 的峰形及偶合常 数说明它们均位于椅式构象的平伏键, 分别取 $\alpha$ 和 $\beta$ 方 向. NOESY 相关谱中(图 3), Me-19 与 H-1a, H-4b 相关, H-9 与 H-1b, H-5 相关, 表明 H-5 与 Me-19 不同面, H-5 为 $\alpha$ 取向, $\mathrm{A} / \mathrm{B}$ 环为反式稠合. 由此确定了化合物 $\mathbf{2}$ 的结 构, 并被命名为 5-epikarelinine.

化合物 3 为无色晶体, 易溶于氯仿. 其 HRESIMS $\left(\mathrm{m} / \mathrm{z} 414.3362[\mathrm{M}+\mathrm{H}]^{+}\right.$, 计算值为 414.3367)显示其分子 式为 $\mathrm{C}_{27} \mathrm{H}_{43} \mathrm{NO}_{2}$, 具有 7 个不饱和度. 其 ${ }^{1} \mathrm{H}$ NMR 谱数据 (表 1)显示 1 个单峰甲基, 2 个双峰甲基, 以及 2 个连氧
次甲基信号. 其 ${ }^{13} \mathrm{C} N \mathrm{NMR}$ 谱(表 1)中显示化合物 3 有 27 个碳信号, 结合 DEPT 和 HSQC 谱, 可以归属为 3 个甲 基, 11 个亚甲基(包含 2 个连氮亚甲基 $\delta_{\mathrm{C}} 57.2, \mathrm{C}-18 ; 60.0$, C-26), 10 个次甲基(包含 2 个连氧次甲基 $\delta_{\mathrm{C}} 72.1, \mathrm{C}-3$; $73.3, \mathrm{C}-6), 1$ 个季碳 $\left(\delta_{\mathrm{C}} 35.6, \mathrm{C}-10\right)$, 以及 1 个双键信号 $\left(\delta_{\mathrm{C}} 128.2, \mathrm{C}-13 ; 130.8, \mathrm{C}-17\right)$. 根据以上核磁信号推断化 合物 3 为 cevanine 骨架的异甾体生物碱 ${ }^{[15]}$. 对比该化合 物与已知化合物 ebeienine (4) ${ }^{[16]}$ 的核磁数据, 发现二者 具有相同的 $A \sim E$ 环结构, 区别在于 $F$ 环, 推测可能是 $F$ 环的 27 位甲基构型发生了变化. 比较化合物 $\mathbf{3}$ 与具有 $27 \beta$ 甲基的 cevanine 型生物碱 ebeinine $\mathrm{e}^{[15]}$ 的核磁数据, 发 现它们 $\mathrm{F}$ 环的碳信号一致，证实了化合物 3 的 27 位甲基 为 $\beta$ 取向. 分析化合物 3 的 ${ }^{1} \mathrm{H}-{ }^{-1} \mathrm{H}$ COSY 与 $\mathrm{HMBC}$ 相关 谱(图 2), 并通过 $X$ 射线单晶衍射(图 4), 确定了化合物 $\mathbf{3}$ 的结构及绝对构型, 并命名为 27-epiebeienine.

此外，本文还分离鉴定了 3 个已知化合物，将 NMR 和 MS 数据与文献对比, 分别鉴定为 ebeienine $(4)^{[16]}$, persicanidine $\mathrm{B}(5)^{[17]}$ 和 heilonine $(6)^{[18]}$, 其中化合物 $\mathbf{6}$ 的 绝对构型也经 $X$ 射线单晶衍射得以确证. 化合物 $4 \sim 6$ 均为首次从该植物中分离得到.

植物来源的 jervine 型异甾体生物碱多为 $5 \alpha$-jervine 型, $\mathrm{A} / \mathrm{B}$ 环反式稠合. 已报道的少量 $5 \beta$-jervine 型生物碱

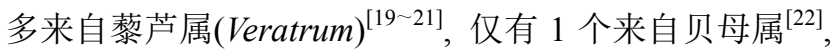
化合物 1 是贝母属中发现的第 2 例此类型生物碱, 具有 $\mathrm{A} / \mathrm{B}$ 环顺式稠合方式. 此外, 15 位羟基取代的 jervine 型 生物碱也较为少见 ${ }^{[21,22]}$, 化合物 $\mathbf{1}$ 和 $\mathbf{2}$ 是贝母属中首次 发现的具有 $15 \alpha-\mathrm{OH}$ 取代的 jervine 型生物碱. 化合物 3 是少见的具有 $\Delta^{13(17)}$ 双键的 cevanine 型异甾体生物碱, 仅有 3 个此类化合物被报道 ${ }^{[15,16,23]}$.

由于在提取生物碱的过程中使用了酸碱处理, 且化 合物 1 和 2 的结构中存在 6-酮, H-5 可能比较活泼, 在实 验条件下有可能异构化. 为了验证或排除这种可能性, 我们模拟实验条件, 对化合物 $\mathbf{2}$ 进行了酸碱处理, HPLC 分析发现化合物 2 在酸碱处理之后结构没有变化; 同时 对砂贝母的 $90 \%$ 乙醇提取物未作酸碱处理，直接进行 ODS (ostade-cylsilane) 反相硅胶柱色谱, 制备 60\%甲醇 部位. HPLC 分析发现化合物 $\mathbf{1}$ 和 $\mathbf{2}$ 在此部位中天然存 在. 因此, 化合物 1 和 2 中 6-酮的存在, 在目前的酸碱 条件下, 不会导致 H-5 的异构化, 两个化合物均为天然 产物.

\section{2 实验部分}

\section{1 仪器与试剂}

Rudolph Autopol IV-T 旋光仪(美国 Rudolph 公司); Nicolet iS5 傅里叶变换红外光谱仪(美国 Thermo Fisher 
Scientific 公司); Agilent 1100 LC/MSD-SL (美国 Agilent Technologies 公司)和 AB 5600+Q TOF 质谱仪(美国 AB SCIEX 公司); Varian Mercury Plus $400 \mathrm{MHz}$ (美国 Varian 公司)和 Bruker Avance $600 \mathrm{MHz}$ 核磁共振仪(德国 Bruker 公司); Shimadzu Essentia LC-16 高效液相色谱仪 (日本 Shimadzu 公司); Luna ODS column $(250 \mathrm{~mm} \times 10$ $\mathrm{mm}, 5 \mu \mathrm{m}$ )液相色谱柱(美国 Phenomenex 公司); ODS 反 相硅胶 (50 $\mu \mathrm{m}$, 日本 YMC 公司); Sephadex LH-20 (美国 $\mathrm{GE}$ 生物医药); 正相柱色谱胶(200 300 目)和薄层色谱 HSGF254 硅胶预制板(烟台芝眾黄务硅胶开发试验厂). 分析纯甲醇、乙醇、二氯甲烷和三氯甲烷等溶剂(国药 集团化学试剂有限公司); HPLC 级甲醇、乙腈 (Adamas-beta 公司).

砂贝母干燥鳞茎样品于 2017 年 6 月采自新疆伊犁 地区. 经新疆药物研究所何江研究员鉴定为贝母属植物 砂贝母 (Fritillaria karelinii Baker)的鳞茎, 凭证标本 (TCM 2017-06 Hou)保存于复旦大学药学院生药学教研 室.

\section{2 提取与分离}

将干燥的砂贝母鳞茎 $(1.85 \mathrm{~kg})$ 粉碎后于室温下用 $90 \%$ 乙醇 $(60 \mathrm{~L})$ 进行渗漉提取, 减压浓缩合并得到浸膏 $28 \mathrm{~g}$. 将浸膏混悬于水中, 加 $5 \%$ 酒石酸调 $\mathrm{pH}$ 至 2 , 用二 氯甲烷萃取脱脂; 酸水层加氨水调 $\mathrm{pH}$ 至 11 , 用三氯甲 烷萃取. 将三氯甲烷萃取物( $3.2 \mathrm{~g})$ 进行正相硅胶柱色谱 分离, 以二氯甲烷一甲醇 $(V: V=30: 1 \rightarrow 20: 1 \rightarrow 15$ : $1 \rightarrow 10: 1 \rightarrow 6: 1 \rightarrow 3: 1 \rightarrow 0: 1)$ 进行梯度洗脱, TLC 检 测合并流份, 得到组分 Fr.1 Fr.5. 其中 Fr.2 $(75 \mathrm{mg})$ 经 ODS 柱色谱(甲醇-水, $V: V=50: 50 \rightarrow 60: 40 \rightarrow 70$ : $30 \rightarrow 80: 20 \rightarrow 90: 10 \rightarrow 100: 0$ ), 再经半制备 HPLC(甲 醇-水, $V: V=86: 14)$ 纯化得到化合物 $3\left(10.0 \mathrm{mg}, t_{\mathrm{R}} 18\right.$ $\mathrm{min})$ 和 $4\left(7.2 \mathrm{mg}, t_{\mathrm{R}} 25.5 \mathrm{~min}\right)$. Fr.3 $(137 \mathrm{mg})$ 经过 Sephadex LH-20 (甲醇)柱色谱分离, 得到组分 Fr.3.1 Fr.3.3. 其中 Fr.3.2 (27 mg) 和 Fr.3.3 (34 mg)经半制备 HPLC 纯化, 分别得到化合物 1 (甲醇-水, $V: V=52$ : $48,7.2 \mathrm{mg}, t_{\mathrm{R}} 12 \mathrm{~min}$ ) 和 2 (甲醇-水, $V: V=50: 50,5.0$ $\mathrm{mg}, t_{\mathrm{R}} 17 \mathrm{~min}$ ). Fr.4 (274 mg)经过 Sephadex LH-20 (甲醇) 柱色谱分离得到组分 Fr.4.1 Fr.4.6. 其中 Fr.4.2 (32 mg) 和 Fr.4.4 (30 mg)经半制备 HPLC 纯化, 分别得到化合物 5(乙腈-水, $V: V=75: 25,10.0 \mathrm{mg}, t_{\mathrm{R}} 27 \mathrm{~min}$ )和 6 (甲 醇一水, $\left.V: V=82: 18,6.0 \mathrm{mg}, t_{\mathrm{R}} 35 \mathrm{~min}\right)$. HPLC 制备时 的检测波长为 210 和 $254 \mathrm{~nm}$, 流速为 $3 \mathrm{~mL} / \mathrm{min}$.

Karelinine (1): 无色晶体, m.p. $209 \sim 210{ }^{\circ} \mathrm{C}$, 易溶 于甲醇; $[\alpha]_{\mathrm{D}}^{25}-33.0($ c 0.20, $\mathrm{MeOH}) ;{ }^{1} \mathrm{H} \mathrm{NMR}\left(\mathrm{CD}_{3} \mathrm{OD}\right.$, $600 \mathrm{MHz})$ 和 ${ }^{13} \mathrm{C}$ NMR $\left(\mathrm{CD}_{3} \mathrm{OD}, 150 \mathrm{MHz}\right)$ 数据见表 1; IR (KBr) $v_{\max }: 3398,2928,2873,1698,1632,1458,1435$,
1385, 1353, 1285, 1120,1063, 1020, 983, $928 \mathrm{~cm}^{-1}$; (+) ESIMS $m / z: 460.2[\mathrm{M}+\mathrm{H}]^{+} ;(+)$HRESIMS calcd for $\mathrm{C}_{27} \mathrm{H}_{42} \mathrm{NO}_{5}[\mathrm{M}+\mathrm{H}]^{+}$460.3058, found 460.3057 .

5-Epikarelinine (2): 白色无定形粉末，易溶于甲醇; $[\alpha]_{\mathrm{D}}^{25}-15.8$ (c 0.12, MeOH); ${ }^{1} \mathrm{H}$ NMR $\left(\mathrm{CD}_{3} \mathrm{OD}, 600\right.$ $\mathrm{MHz})$ 和 ${ }^{13} \mathrm{C}$ NMR $\left(\mathrm{CD}_{3} \mathrm{OD}, 150 \mathrm{MHz}\right)$ 数据见表 1 ; IR (KBr) $v_{\text {max }}: 3403,2925,2869,1698,1630,1460,1438$, 1380, 1355, 1282, 1124, 1066, 1020, 983, $929 \mathrm{~cm}^{-1}$; (+) ESIMS $m / z: 460.2[\mathrm{M}+\mathrm{H}]^{+} ;(+)$HRESIMS calcd for $\mathrm{C}_{27} \mathrm{H}_{42} \mathrm{NO}_{5}[\mathrm{M}+\mathrm{H}]^{+}$460.3058, found 460.3048 .

27-Epiebeienine (3): 无色晶体, m.p. $243 \sim 244{ }^{\circ} \mathrm{C}$, 易溶于氯仿; $[\alpha]_{\mathrm{D}}^{25}-8.2(c \quad 0.11, \mathrm{MeOH}) ;{ }^{1} \mathrm{H}$ NMR $\left(\mathrm{CDCl}_{3}, 600 \mathrm{MHz}\right)$ 和 ${ }^{13} \mathrm{C} \mathrm{NMR}\left(\mathrm{CDCl}_{3}, 150 \mathrm{MHz}\right)$ 数据见 表 1; IR (KBr) $v_{\text {max }}$ : 3435, 2925, 2853, 1658, 1632, 1440, $1033 \mathrm{~cm}^{-1} ;(+)$ ESIMS $m / z: 414.2[\mathrm{M}+\mathrm{H}]^{+} ;(+)$ HRESIMS calcd for $\mathrm{C}_{27} \mathrm{H}_{44} \mathrm{NO}_{2}[\mathrm{M}+\mathrm{H}]^{+}$414.3367, found 414.3362 .

\subsection{Karelinine (1)和 27-Epiebeienine (3) X 射线单晶 衍射测定}

Karelinine (1) 在二氯甲烷一甲醇-水混合溶剂中，溶 剂挥发得到无色晶体, 采用 Bruker D8 Venture X 射线单 晶衍射仪 $(\mathrm{Ga}-\mathrm{K} \alpha, \lambda=1.34139 \AA)$ 测定. 六边形晶体, $\mathrm{C}_{27} \mathrm{H}_{41} \mathrm{NO}_{5} \cdot \mathrm{H}_{2} \mathrm{O}, \quad M_{\mathrm{r}}=477.62 ; a=16.3741(2) \AA, b=$ 16.3741(2) $\AA, c=16.6637(2) \AA, \alpha=\beta=90^{\circ}, \gamma=120^{\circ} ; V=$ $3869.16(11) \AA^{3}, D_{\mathrm{c}}=1.230 \mathrm{mg} / \mathrm{m}^{3}, Z=6 ; F(000)=1560$, $\mu=0.444 \mathrm{~mm}^{-1}$, 优化方法基于 $F^{2}$ 全矩阵最小二乘法; $T=170.15 \mathrm{~K}$; 空间点群 $P 6_{2}$, 收集衍射点数为 48648 , 独 立衍射点数 $4904[R(\mathrm{int})=0.0481]$; 最后修正值 $R_{1}=$ $0.0316, w R_{2}=0.0773[I>2 \sigma(I)]$; 修正值 $R_{1}=0.0338$, $w R_{2}=0.0791$ (all data); 拟合优度检验 $F^{2}=1.050$; Flack parameter $=0.06(7)$.

27-Epiebeienine (3)在甲醇-水混合溶剂中，溶剂挥 发得到无色晶体, 采用 Bruker D8 Venture $\mathrm{X}$ 射线单晶衍 射仪 (Ga-K $\alpha, \lambda=1.34139 \AA$ ) 测定. 斜方晶, $\mathrm{C}_{27} \mathrm{H}_{43} \mathrm{NO}_{2}$, $M_{\mathrm{r}}=413.62 ; a=10.2408(2) \AA, b=12.0705(2) \AA, c=$ 18.9179(3) $\AA, \alpha=\beta=\gamma=90^{\circ} ; V=2338.47(7) \AA^{3}, D_{\mathrm{c}}=$ $1.175 \mathrm{mg} / \mathrm{m}^{3}, Z=4 ; F(000)=912, \mu=0.357 \mathrm{~mm}^{-1}$, 优化 方法基于 $F^{2}$ 全矩阵最小二乘法; $T=170.02 \mathrm{~K}$; 空间点群 $P 22_{1} 2_{1}$, 收集衍射点数为 27231 , 独立衍射点数 4427 $[R(\mathrm{int})=0.0326]$; 最后修正值 $R_{1}=0.0276, w R_{2}=0.0738$ $[I>2 \sigma(I)]$; 修正值 $R_{1}=0.0279, w R_{2}=0.0741$ (all data); 拟合优度检验 $F^{2}=1.068$; Flack parameter $=0.00(4)$.

化合物 1 和 3 的 CIF 文件存放于英国剑桥 X 射线单 晶衍射数据中心, CCDC 编号分别为 1864999 和 1865000, 具体信息可通过(https://www.ccdc.cam.ac.uk/ 
deposit/)获取.

辅助材料(Supporting Information) 化合物 $1 \sim 3$ 的 MS, 1D/2D NMR, IR 谱图; 化合物 $\mathbf{4} \sim \mathbf{6}$ 的 MS, 1D NMR 谱图; 化合物 1, 3, 6 的 X 射线单晶衍射数据. 这些材料 可以免费从本刊网站(http://sioc-journal.cn/)上下载.

\section{References}

[1] Nanjing University of Chinese Medicine Dictionary of Traditional Chinese Medicine, Shanghai Science and Technology Press, Shanghai, 2005, pp. 296 299, 1274 1275, 3383 3384 (in Chinese).

(南京中医药大学, 中药大辞典, 上海科学技术出版社, 上海, 2005, pp. 296 299, 1274 1275, 3383 3384.)

[2] Chinese Materia Medica Editorial Committee of State Administration of Traditional Chinese Medicine Chinese Materia Medica, Vol. 22, Shanghai Science and Technology Press, Shanghai, 1999, pp. $88 \sim 101$ (in Chinese).

(国家中医药管理局《中华本草》编委会, 中华本草, 第 22 卷, 上 海科学技术出版社, 上海, 1999, pp. 88 101.)

[3] Li, Y.; Yili, A.; Li, J.; Muhamat, A.; Aisa, H. A. Bioorg. Med. Chem. Lett. 2016, 26, 1983.

[4] Wang, D. D.; Wang, S.; Chen, X.; Xu, X. L.; Zhu, J. Y.; Nie, L. H.; Long, X. J. Ethnopharmacol. 2012, 139, 189.

[5] Li, H. J.; Jiang, Y.; Li, P. Nat. Prod. Rep. 2006, 23, 735.

[6] Hao, D. C.; Gu, X. J.; Xiao, P. G.; Peng, Y. Chin. J. Nat. Med. 2013, 11,330 .

[7] Shen, S.; Li, G.; Huang, J.; Chen, C.; Ren, B.; Lu, G.; Tan, Y.; Zhang, J.; Li, X.; Wang, J. Fitoterapia 2012, 83, 785.

[8] Shou, Q. Y.; Tan, Q.; Shen, Z. W. Tetrahedron Lett. 2009, 50, 4185.
[9] Pi, H. F.; Zhang, P.; Ruan, H. L.; Zhang, Y. H.; Sun, H. D.; Wu, J. Z. J. Asian Nat. Prod. Res. 2009, 11, 779.

[10] Lin, B. Q.; Ji, H.; Li, P.; Jiang, Y.; Fang, W. Eur. J. Pharmacol. 2006, 551, 125.

[11] Chen, X. Q.; Mordak, H. V. Flora of China, Vol. 24, Science Press, Beijing, 2000, p. 132.

[12] Pan, X. F.; Zhu, Z. Q. J. Lanzhou Univ. 1979, 24, 54 (in Chinese). (潘金复, 朱子清, 兰州大学学报, 1979, 24, 54.)

[13] Liu, Y. M.; Feng, Y. D.; Lu, X.; Nie, J. B.; Li, W.; Wang, L. N.; Tian, L. J.; Liu, Q. H. Eur. J. Med. Chem. 2017, 137, 280.

[14] Richmond, V.; Garrido Santos, G. A.; Murray, A. P.; Maier, M. S. Steroids 2011, 76, 1160.

[15] Wu, J. Z.; Pan, X. P.; Lou, M. A.; Wang, X. S.; Ling, D. K. Acta Pharm. Sin. 1989, 24, 600 (in Chinese).

(吴继洲, 潘锡平, 娄民安, 王孝生, 凌大奎, 药学学报, 1989, 24, 600.)

[16] Lee, P.; Kitamura, Y.; Kaneko, K.; Shiro, M.; Xu, G. J.; Chen, Y. P.; Hsu, H. Y. Chem. Pharm. Bull. 1988, 36, 4316.

[17] Ori, K.; Mimaki, Y.; Sashida, Y.; Nikaido, T.; Ohmoto, T. Phytochemistry 1992, 31, 3605.

[18] Kitamura, Y.; Nishizawa, M.; Kaneko, K. Tetrahedron 1989, 45, 7281.

[19] Li, Q.; Yang, K. X.; Zhao, Y. L.; Qin, X. J.; Yang, X. W.; Liu, L.; Liu, Y. P.; Luo, X. D. J. Ethnopharmacol. 2016, 179, 274.

[20] Cong, Y.; Li, J.; Zhang, Q. C.; Guo, J. G.; Li, S. S. Helv. Chim. Acta 2015, 98,85 .

[21] Zhou, X. F.; Gao, Z. G.; Han, X. R.; Zhao, W. J.; Wang, S. S. Chin. Chem. Lett. 2010, 21, 1209.

[22] Du, Y.; Zheng, Z. G.; Yu, Y.; Wu, Z. T.; Liang, D.; Li, P.; Jiang, Y.; Li, H. J. J. Pharm. Biomed. Anal. 2017, 142, 201.

[23] Atta-ur-Rahman; Farooq, A.; Choudhary, M. I.; Gilani, A. H.; Shaheen, F.; Ali, R. A.; Noor-e-ain, F.; Sener, B. Planta Med. 1994, 60, 377. 